\title{
Single-cell reconstruction of the human early maternal-fetal interface
}

\section{$\underline{\text { Authors }}$}

Authors: Roser Vento-Tormo ${ }^{+1,2}$, Mirjana Efremova ${ }^{\star 1}$, Rachel A. Botting, Margherita Y. Turco ${ }^{24,5}$, Miquel Vento-Tormo 6, Kerstin B. Meyer', Jongeun Park', Emily Stephenson’, Krzysztof Polański', Angela Goncalves ${ }^{17}$, Lucy Gardner ${ }^{24}$, Staffan Holmqvist', Johan Henriksson', Angela Zou', Andrew M. Sharkey ${ }^{24}$, Ben Millar ${ }^{3}$, Barbara Innes ${ }^{3}$, Laura Wood', Anna Wilbrey-Clark', Rebecca P. Payne ${ }^{3}$, Martin A. Ivarsson, Steve Lisgo', Andrew Filby ${ }^{3}$, David H. Rowitch', Judith N. Bulmer', Gavin J. Wright', Michael J. T. Stubbington', Muzlifah Haniffa ${ }^{13,10^{*}}$, Ashley Moffett ${ }^{24^{4}}$, Sarah A. Teichmann ${ }^{1,1,12^{2 *}}$

\section{Affiliations:}

1 Wellcome Sanger Institute, Wellcome Genome Campus, Hinxton, Cambridge CB10 1SA, United Kingdom

2 Centre for Trophoblast Research, University of Cambridge, UK

3 Institute of Cellular Medicine, Newcastle University, Newcastle upon Tyne, NE2 4HH, UK.

4 Department of Pathology, University of Cambridge, Cambridge, CB2 1QP, UK.

5 Department of Physiology, Development and Neuroscience, University of Cambridge, Cambridge, CB2 3EL, UK

6 YDEVS software development, Valencia 46009, Spain

7 German Cancer Research Center (DKFZ), Heidelberg, Germany

8 Department of Paediatrics, University of Cambridge, Cambridge CB2 0QQ, UK.

9 Human Developmental Biology Resource, Institute of Genetic Medicine, Newcastle University, Newcastle upon Tyne, NE1 3BZ

10 Department of Dermatology and NIHR Newcastle Biomedical Research Centre, Newcastle Hospitals NHS Foundation Trust, Newcastle upon Tyne NE2 4LP, UK

11 Theory of Condensed Matter Group, The Cavendish Laboratory, University of Cambridge, JJ Thomson Ave, Cambridge CB3 0EH, UK.

12 European Molecular Biology Laboratory, European Bioinformatics Institute (EMBL-EBI), Hinxton, Cambridge CB10 1SD, UK.

These authors contributed equally.

JJoint last and corresponding authors: st9@sanger.ac.uk, am485@cam.ac.uk, m.a.haniffa@newcastle.ac.uk

keywords: cell-cell communication, single-cell RNA-seq, early pregnancy, decidua, placenta, maternal-fetal interface, NK cells, trophoblast cells, stromal cells 
During early human pregnancy, the fetal placenta implants into the uterine mucosa (decidua) where placental trophoblast cells intermingle and communicate with maternal cells. Trophoblastdecidual interactions underlie common diseases of pregnancy including pre-eclampsia and stillbirth. Here, we profile transcriptomes of $\sim 70,000$ single cells from first trimester placentas with matched maternal blood and decidual cells. The cellular composition of human decidua reveals new subsets of perivascular and stromal cells, which are located in distinct decidual layers. There are three major subsets of decidual NK cells, with distinctive immunomodulatory and chemokine profiles. We develop a repository of ligand-receptor complexes (https://cellphonedb.org/) and a statistical tool to predict the cell-type specificity of cell-cell communication via these molecular interactions. This identifies many regulatory interactions that prevent any damaging innate or adaptive immune responses in this environment. Our single cell atlas of the maternal-fetal interface reveals the cellular organization and interactions critical for placentation and reproductive success.

During early pregnancy, the uterine mucosal lining, the endometrium, is transformed into decidua under the influence of progesterone. Decidualisation results from a complex and well-orchestrated differentiation program that involves all cellular elements of the mucosa: stromal, glandular, and immune cells, including the distinctive decidual Natural Killer cells $(\mathrm{dNK})^{1,2}$. The blastocyst implants into the decidua and initially, before arterial connections are established, uterine glands are the source of histotrophic nutrition in the placenta ${ }^{3,4}$. Following implantation, placental extravillous trophoblast cells (EVT) invade through the decidua and move towards the spiral arteries, where they destroy the smooth muscle media and transform the arteries into high conductance vessels ${ }^{5}$. Balanced regulation of EVT invasion is critical to pregnancy success: arteries must be sufficiently transformed, but excessive invasion prevented, to ensure correct allocation of resources to both mother and baby ${ }^{6}$. The pivotal regulatory role of the decidua is obvious from the life-threatening, uncontrolled, trophoblast invasion that occurs when the decidua is absent as when the placenta implants on a previous cesarean section scar ${ }^{7}$.

EVT have a unique HLA profile: they do not express the dominant T cell ligands, class I HLA-A and HLA-B or class II molecules ${ }^{8,9}$, but do express HLA-G and HLA-E and polymorphic HLA-C class I molecules. These trophoblast HLA ligands have receptors expressed by the dominant decidual immune cells, dNK, including maternal killer immunoglobulin-like receptors (KIR), that bind HLA-C molecules ${ }^{10,11}$. Certain combinations of maternal KIR and fetal HLA-C genetic variants are associated with pregnancy disorders such as pre-eclampsia, where trophoblast invasion is deficient ${ }^{12}$. However, detailed understanding of the cellular interactions in the decidua supporting early pregnancy is lacking. In this study, we used single-cell transcriptomics to comprehensively resolve cell states involved in maternal-fetal communication in the decidua during early in pregnancy when the placenta is established. We then used a computational framework to predict cell-type specific ligand-receptor complexes and present a new database: CellPhoneDB.org. By integrating these predictions with spatial 
in situ analysis, we construct a detailed molecular and cellular map of the human decidual-placental interface.

\section{Results}

\section{Maternal and fetal cells in early pregnancy}

We combined droplet-based encapsulation (10x Genomics Chromium) ${ }^{13}$ and plate-based Smart-seq2 (SS2) ${ }^{14}$ single cell transcriptome profiles from the maternal-fetal interface (eleven decidua and five placenta from 6-14 gestational weeks) and six matched peripheral blood mononuclear cells (PBMC) (Fig. 1a-b, Supplementary Table 1-2, Extended Data Fig. 1). After computational quality control and integration of transcriptomes from both technologies, we performed graph-based clustering (see Methods) of the combined dataset and used cluster-specific marker genes to annotate the clusters (Fig. 1c, Extended Data Fig. 2-3, Supplementary Table 2). We studied T cell composition and clonal expansion using full-length transcriptomes from SS2 and reconstructed the T-cell receptor (TCR) sequences from this data showing CD8 T cell expansion in the decidua (Fig. 1d).

We aligned scRNA-seq reads from each cell with overlapping single nucleotide polymorphisms (SNPs) called from maternal and fetal genomic DNA to assign cells as fetal or maternal (Fig. 1g, Extended Data Fig. 3e). As expected, decidual samples contained mostly maternal cells with a few fetal $H L A-G^{+}$ EVT. Fetal cells dominate the placental samples, with the exception of maternal macrophages (M3 cluster) that express $C D 14, S 100 A 9, C D 163, C D 68, C S F 1 R$. These are probably derived from blood monocytes incorporated into the syncytium (Extended Data Fig. 3f) ${ }^{15}$.

\section{Cell communication predicted by CellPhoneDB}

To systematically study the interactions between fetal and maternal cells in the decidual-placental interface, we developed 'CellPhoneDB.org', a repository of ligand-receptor interacting pairs that accounts for their subunit architecture, representing heteromeric complexes accurately (Extended Data Fig. 4a). Both secreted and cell surface molecules are considered, so this encompasses ligand-receptor interactions mediated by diffusion of secreted molecules. Our repository forms the basis of a computational approach to identify biologically relevant ligand-receptor complexes. We consider the expression levels of ligands and receptors within each cell type, and using empirical shuffling, calculate which ligand-receptor pairs display significant cell type specificity (Extended Data Fig. 4b, see Methods). This predicts molecular interactions between cell populations via specific protein complexes, and generates a potential cell-cell communication network in the decidua and placenta (Extended Data Fig. 4c-e, Supplementary Table 3-4).

\section{Trophoblast differentiation by scRNAseq}


To investigate maternal-fetal interactions at the decidual-placental interface we first analysed fetal trophoblast cells isolated from placental and decidual samples; the latter contain invasive EVT (Extended Data Fig. 5a-b). Consistent with previous results ${ }^{16,17}$, we resolved two distinct trophoblast differentiation pathways (Fig. 2a). As expected, decidual EVT are at the end of the trajectory, have high $H L A-G$ expression, and no longer express cell cycle genes (Extended Data Fig. 5c). In villous cytotrophoblasts (VCT), CellPhoneDB predicts interactions between receptors involved in cellular proliferation and differentiation (EGFR, NRP2 and MET) with $H B E G F$ expressed by Hofbauer cells, and $P G F$ and $H G F$ expressed by different placental fibroblast subsets (Supplementary Table 5, Fig. 2b).

In contrast, during EVT differentiation, there is upregulation of receptors involved in immunomodulation, cellular adhesion and invasion, whose ligands are expressed by decidual cells (Fig. $2 b)$. For example, $A C K R 2$ is a decoy receptor for inflammatory cytokines produced by maternal immune cells $^{18}$ and $C X C R 6$ is a chemokine receptor binding CXCL16 expressed by the maternal macrophages. Expression of $T G F \beta$ and its receptor, whose function is to suppress immune responses ${ }^{19}$ and activate epithelial-mesenchymal transition (EMT), increases as EVT differentiate. Components involved in the EMT program are upregulated at the end of the trajectory ${ }^{20}$ (Extended Data Fig. 5d); these include metalloproteinases PAPPA and PAPPA2, known to be involved in cellular invasion. In pregnancy, decreased PAPPA levels is a biomarker for disorders of pregnancy associated with defective EVT invasion (pre-eclampsia and fetal growth restriction) ${ }^{21}$.

\section{Stromal cells in the two decidual layers}

EVT initially invade through the surface epithelium into the decidua compacta. Beneath this is the decidua spongiosa that contains hypersecretory glands providing histotrophic nutrition to the early conceptus. Markers distinguishing the different decidual fibroblast populations identify two clusters of perivascular cells, PV1/PV2, that share expression of the smooth muscle marker $(M G P)$ and are distinguished by different levels of MCAM and MMP11 (higher in PV1 and PV2 respectively) (Fig. 3a, Supplementary Table 6). There are three clusters of stromal cells, dS1/dS2/dS3, all expressing the WNT inhibitor DKK1. dS1 shares the expression of $A C T A 2$ and $T A G L N$ with PV1/PV2 and lacks expression of classical decidual markers prolactin ( $P R L)$ and IGFBPI. In contrast, dS2/dS3, express $I G F B P 1 / 2 / 6$ and share markers with two recently described in vitro decidualised stromal cell subsets ${ }^{22}$. The dS 3 subset expresses $P R L$, as well as genes involved in steroid biosynthesis (e.g. CYP11A1) (Extended Data Fig. 6).

To locate the different perivascular and stromal populations in situ, we used immunohistochemistry (IHC) and multiplexed single molecule fluorescent in situ hybridisation (smFISH) for selected markers on serial sections of decidua parietalis. These experiments confirm that cells expressing ACTA2 and MCAM are present in the media of the spiral arteries ${ }^{23}$ and show that MMP11 is also present, 
demonstrating that both PV1 and PV2 are perivascular (Fig. 3b, Extended Data Fig. 7). ACTA2 ${ }^{+} \mathrm{dS} 1$ stromal cells are present between glands in decidua spongiosa whilst $I G F B P 1^{+}$and $P R L^{+} \mathrm{dS} 2 / \mathrm{dS} 3$ cells are located in decidua compacta (Fig. 3c-d, Extended Data Fig. 7). CYP11A1 is also expressed more abundantly in decidua compacta compared to spongiosa (Extended Data Fig. 6b).

Our CellPhoneDB tool predicts that the cognate receptors for angiogenic factors expressed by PV1/2 (e.g. ANGPT1, VEGFA) are located in endothelium (Fig. 3e). EVT first invade the decidua compacta where $\mathrm{dS} 2 / 3$ express high levels of $L G A L S 9$ and $C L E C 2 D$. These molecules could interact with their inhibitory receptors, TIM3 and KLRB1, expressed by dNK subsets, enabling the stroma to suppress inflammatory reactions in the decidua.

\section{Three decidual NK cell states}

We identified three main dNK subsets which all co-express the tissue-resident markers, CD49a and CD9 (Extended Data Fig. 8). dNK1 express CD39, CYP26A1 and B4GALNT1 while the defining markers of dNK2 cells are $A N X A 1$ and ITGB2; the latter is shared with dNK3 (Fig. 4a, Supplementary Table 7). dNK3 cells express CD160, KLRB1 and CD103, but not the ILC marker CD127 (Extended Data Fig. 8a).

KIR genes are polymorphic and highly homologous making quantification of mRNA expression of individual KIR genes challenging ${ }^{12}$. We therefore developed KIRid, a method that uses full-length transcript SS2 data to map each donor's single cell reads to the corresponding donor-specific reference of KIR alleles (see Methods). We find that dNK1 cells express higher levels of KIR that can bind to HLA-C molecules: activating KIR2DS1/S4 and inhibitory KIR2DL1/L2/L3 (Fig. 4b-c, Supplementary Table 8). LILRB1, the receptor for the dimeric form of HLA-G molecules, is only expressed by the dNK1 subset. Both dNK1 and dNK2, but not dNK3, express activating (NKG2C,NKG2E) and inhibitory $(N K G 2 A)$ receptors for HLA-E molecules (Fig. 4c). These results predict a likely function of dNK1 in the recognition and response to EVT.

To investigate these three dNK populations further, we analysed six decidual samples by flow cytometry using CD49a (resident $\mathrm{dNK}$ ), combined with markers for each dNK subset predicted from our transcriptomics data (CD39, ITGB2, CD103, KIR2DL1) (Fig. 4d). We confirmed the presence of the three dNK populations by flow cytometry and the preferential expression of KIR2DL1 in dNK1 (Fig. 4d, Supplementary Table 9). We analysed the morphology of dNK subsets by Giemsa staining of cells isolated by flow cytometric sorting (Extended Data Fig. 8c). dNK1 contain more cytoplasmic granules than dNK2 and dNK3, consistent with our scRNAseq data showing higher levels of PRF, GNLY, GZMA, GZMB RNA expression in this subset (Fig. 4e). Higher levels of expression of the granule proteins (PRF1, GNLY, GZMA and GZMB) are found in $\mathrm{KIR}^{+}$compared to KIR- dNK cells by flow cytometry (Fig. 4f). dNK1 also express high levels of enzymes involved in glycolysis (Fig. 4g). Thus, the features of $\mathrm{dNK} 1$ are: active glycolytic metabolism, higher expression of KIR, LILRB1 and cytoplasmic granule proteins, suggesting that it is dNK1 that particularly interact with EVT. 
First pregnancies are associated with lower proportions of dNK cells expressing LILRB1 ${ }^{24}$, lower birth weights, and increased occurrence of disorders such as pre-eclampsia ${ }^{25}$. Metabolomic programming of mature "memory" NK cells also occurs in chronic human cytomegalovirus (HCMV) infection ${ }^{26}$. Together, these findings are consistent with 'priming' of dNK1 during first pregnancy so they can respond more effectively to the implanting placenta in subsequent pregnancies.

\section{Immunomodulation during early pregnancy}

We next used CellPhoneDB to identify expression of cytokines and chemokines by dNK and predict their interactions with other cells at the maternal-fetal interface (Extended Data Fig. 9a, Fig. 5a). We find no evidence, however, for significant $V E G F A$ or $I F N G$ expression by dNK in vivo ${ }^{24,27}$, probably because these previous studies used dNK cells cultured with IL-2 or IL-15 in vitro.

dNK1 cells express higher levels of CSF1 whose receptor, CSF1R, is expressed by EVTs and macrophages (Fig. 5a-b). Secretion of CSF1 by dNK cells, and interaction with the CSF1R on EVT have been previously described ${ }^{28,29}$, and we now pinpoint this interaction specifically to the dNK1 subset. In contrast, dNK2-3 express high levels of XCL1 and CCL5 is highly expressed by dNK3 (Fig. 5a-b, Extended Data Fig. 9b). The receptor for CCL5, CCR1, is expressed by EVT, suggesting a role for dNK3 in regulating EVT invasion ${ }^{30}$. The expression pattern of XCL1-XCR1 ligand-receptor complex suggests functional interactions between $\mathrm{dNK} 2 / 3$ and both EVT and DC1. NK cell-mediated DC1 recruitment to induce $\mathrm{T}$ cell anergy occurs in tumour microenvironments ${ }^{31}$. We find an increased proportion of $\mathrm{DC} 1$ compared to $\mathrm{DC} 2$, possibly leading to decidual $\mathrm{CD} 8^{+} \mathrm{T}$ cell expansion (Fig. 1d), but coexpression of $P D 1$ suggests that local T cell activation is limited.

Our results collectively suggest that in the decidua microenvironment all damaging maternal $\mathrm{T}$ or NK responses to fetal trophoblasts are prevented. There is high expression of PDL1 in EVT, confirmed in situ using IHC on serial sections of decidua basalis (site of trophoblast invasion) stained for PDL1 and HLA-G (Extended Data Fig. 9c). We also identify putative inhibitory interactions between dNK and EVT, in addition to the previously discussed KIR2DL1/2/3 and HLA-C receptor-ligand complexes. These include KLRB1 and TIGIT highly expressed by dNK3, potentially binding CLEC2D and PVR, expressed by EVT (Fig. 5a).

We predict that the decidua immune microenvironment prevents inflammatory responses potentially triggered by trophoblast invasion and medial destruction of the spiral arteries by trophoblast (Fig. 5c). Macrophage subsets $(\mathrm{dM})$ expresses immunomodulatory molecules like IL10 whose receptor is expressed by EVT and maternal endothelial, stromal and myeloid cells. dNK1 cells and dNK2/3 express high levels of SPINK2 and ANXA1 respectively. Both have anti-inflammatory roles such as inhibiting kallikreins $^{32}$. The dNK1 subset expresses $C D 39$, which together with $C D 73$ converts ATP to adenosine to prevent immune activation ${ }^{33}$ (Fig 5c, Extended Data Fig. 9b). Expression of $C D 73$ is high in epithelial glands and EVT, and the adenosine receptor (ADORA3) is present in macrophages (Fig.5c, Extended Data Fig. 9b). KIR2DL1 ${ }^{+}$dNK1 are in close physical contact with HLA-G ${ }^{+}$EVT (Extended Data Fig. 
9d), suggesting they could together convert extracellular ATP, an inflammatory signal released upon cell death, to adenosine ${ }^{34}$.

\section{Discussion}

Reproductive success depends on events occurring during placentation in the first-trimester decidua ${ }^{35}$. Other scRNAseq studies of uterine cells in pregnancy have analysed cells at the end of gestation ${ }^{16,36}$ or are restricted to fetal placental populations ${ }^{17}$. Ours is the first comprehensive single-cell transcriptomics atlas of the maternal-fetal interface between 6-14 weeks of gestation (Extended Data Fig. 10). Similar to previous scRNAseq analyses ${ }^{36-39}$, we predict possible ligand-receptor interactions, and have developed a new open repository for this purpose: CellPhoneDB.org. This database accounts for the multimeric nature of ligands and receptors and is integrated with a statistical framework that predicts enriched cellular interactions between two cell types.

We show the differentiation trajectory of trophoblast cells to either SCT (nutrient exchange) or EVT (invade and remodel the spiral arteries) and predict the ligand-receptor interactions likely to control these processes. Our findings also suggest an environment where any adaptive or innate immune responses damaging to the placenta or to the uterus are minimised. This is critical for the peaceful compromise needed to define the territorial boundary between mother and fetus. The environment has striking parallels with that around tumours, where inflammatory and adaptive immune responses are also dampened ${ }^{40}$. $\mathrm{dNK}$ cells comprise $\sim 70 \%$ of immune cells in first trimester decidua ${ }^{41,42}$ and we now define three major $\mathrm{dNK}$ subsets and predict that their likely function is to mediate the extent of trophoblast invasion in addition to coordinating multiple immunomodulatory pathways involving myeloid cells, $\mathrm{T}$ cells and stromal cells. Maternal immune responses are restrained by diverse classes of signalling molecules: cell surface expression of checkpoint inhibitors such as PD1/PDL1 or TIGIT, tethered ligand-receptor complexes, secreted proteins, as well as small molecules (e.g. adenosine and steroid hormones). We also show that the dNK1 express receptors for trophoblast HLA-C and HLA-G molecules, and can be primed metabolically through increased expression of glycolytic enzymes. Their increased expression of glycolytic enzymes (metabolic priming) suggests they could be responsible for the different reproductive outcomes found in first compared to subsequent pregnancies

In summary, we identify many novel molecular and cellular mechanisms that are operating to generate a physiologically peaceful decidual environment. This cell atlas of the early maternal-fetal interface provides an essential resource for understanding normal and pathological pregnancies. 
Online content Any Methods, including any statements of data availability and Nature Research reporting summaries, along with any additional references and Source Data files, are available in the online version of the paper at

1. Ramathal, C. Y., Bagchi, I. C., Taylor, R. N. \& Bagchi, M. K. Endometrial decidualization: of mice and men. Semin. Reprod. Med. 28, 17-26 (2010).

2. Koopman, L. A. et al. Human decidual natural killer cells are a unique NK cell subset with immunomodulatory potential. J. Exp. Med. 198, 1201-1212 (2003).

3. Burton, G. J., Watson, A. L., Hempstock, J., Skepper, J. N. \& Jauniaux, E. Uterine glands provide histiotrophic nutrition for the human fetus during the first trimester of pregnancy. J. Clin. Endocrinol. Metab. 87, 2954-2959 (2002).

4. Hempstock, J., Cindrova-Davies, T., Jauniaux, E. \& Burton, G. J. Endometrial glands as a source of nutrients, growth factors and cytokines during the first trimester of human pregnancy: a morphological and immunohistochemical study. Reprod. Biol. Endocrinol. 2, 58 (2004).

5. Burton, G. J., Woods, A. W., Jauniaux, E. \& Kingdom, J. C. P. Rheological and physiological consequences of conversion of the maternal spiral arteries for uteroplacental blood flow during human pregnancy. Placenta 30, 473-482 (2009).

6. Fisher, S. J. Why is placentation abnormal in preeclampsia? Am. J. Obstet. Gynecol. 213, S115$22(2015)$.

7. Jauniaux, E. \& Burton, G. J. Placenta accreta spectrum: a need for more research on its aetiopathogenesis. BJOG 125, 1449-1450 (2018).

8. Apps, R., Gardner, L. \& Moffett, A. A critical look at HLA-G. Trends Immunol. 29, 313-321 (2008).

9. Apps, R. et al. Human leucocyte antigen (HLA) expression of primary trophoblast cells and placental cell lines, determined using single antigen beads to characterize allotype specificities of anti-HLA antibodies. Immunology 127, 26-39 (2009).

10. Sharkey, A. M. et al. Killer Ig-like receptor expression in uterine NK cells is biased toward recognition of HLA-C and alters with gestational age. J. Immunol. 181, 39-46 (2008).

11. Parham, P. \& Moffett, A. Variable NK cell receptors and their MHC class I ligands in immunity, 
reproduction and human evolution. Nat. Rev. Immunol. 13, 133-144 (2013).

12. Moffett, A. \& Colucci, F. Co-evolution of NK receptors and HLA ligands in humans is driven by reproduction. Immunol. Rev. 267, 283-297 (2015).

13. Zheng, G. X. Y. et al. Massively parallel digital transcriptional profiling of single cells. Nat. Commun. 8, 14049 (2017).

14. Picelli, S. et al. Full-length RNA-seq from single cells using Smart-seq2. Nat. Protoc. 9, 171 (2014).

15. Burton, G. J. \& Watson, A. L. The Structure of the Human Placenta: Implications for Initiating and Defending Against Virus Infections. Rev. Med. Virol. 7, 219-228 (1997).

16. Tsang, J. C. H. et al. Integrative single-cell and cell-free plasma RNA transcriptomics elucidates placental cellular dynamics. Proc. Natl. Acad. Sci. U. S. A. 114, E7786-E7795 (2017).

17. Liu, Y. et al. Single-cell RNA-seq reveals the diversity of trophoblast subtypes and patterns of differentiation in the human placenta. Cell Res. (2018). doi:10.1038/s41422-018-0066-y

18. Madigan, J. et al. Chemokine scavenger D6 is expressed by trophoblasts and aids the survival of mouse embryos transferred into allogeneic recipients. J. Immunol. 184, 3202-3212 (2010).

19. Mariathasan, S. et al. TGF $\beta$ attenuates tumour response to PD-L1 blockade by contributing to exclusion of T cells. Nature 554, 544-548 (2018).

20. Maltepe, E. \& Fisher, S. J. Placenta: the forgotten organ. Annu. Rev. Cell Dev. Biol. 31, 523-552 (2015).

21. Bolnick, J. M. et al. Altered Biomarkers in Trophoblast Cells Obtained Noninvasively Prior to Clinical Manifestation of Perinatal Disease. Sci. Rep. 6, 32382 (2016).

22. Lucas, E. S. et al. Reconstruction of the Decidual Pathways in Human Endometrial Cells Using Single-Cell RNA-Seq. bioRxiv 368829 (2018). doi:10.1101/368829

23. Muñoz-Fernández, R. et al. Human predecidual stromal cells have distinctive characteristics of pericytes: Cell contractility, chemotactic activity, and expression of pericyte markers and angiogenic factors. Placenta 61, 39-47 (2018).

24. Gamliel, M. et al. Trained Memory of Human Uterine NK Cells Enhances Their Function in Subsequent Pregnancies. Immunity 48, 951-962.e5 (2018).

25. Kozuki, N. et al. The associations of parity and maternal age with small-for-gestational-age, 
preterm, and neonatal and infant mortality: a meta-analysis. BMC Public Health 13 Suppl 3, S2 (2013).

26. Cichocki, F. et al. ARID5B regulates metabolic programming in human adaptive NK cells. $J$. Exp. Med. (2018). doi:10.1084/jem.20172168

27. Hanna, J. et al. Decidual NK cells regulate key developmental processes at the human fetalmaternal interface. Nat. Med. 12, 1065-1074 (2006).

28. Jokhi, P. P., King, A., Boocock, C. \& Loke, Y. W. Secretion of colony stimulating factor-1 by human first trimester placental and decidual cell populations and the effect of this cytokine on trophoblast thymidine uptake in vitro. Hum. Reprod. 10, 2800-2807 (1995).

29. Hamilton, G. S., Lysiak, J. J., Watson, A. J. \& Lala, P. K. Effects of colony stimulating factor-1 on human extravillous trophoblast growth and invasion. J. Endocrinol. 159, 69-77 (1998).

30. Sato, Y. et al. Trophoblasts acquire a chemokine receptor, CCR1, as they differentiate towards invasive phenotype. Development 130, 5519-5532 (2003).

31. Böttcher, J. P. et al. NK Cells Stimulate Recruitment of $\mathrm{cDC} 1$ into the Tumor Microenvironment Promoting Cancer Immune Control. Cell (2018). doi:10.1016/j.cell.2018.01.004

32. Sotiropoulou, G. \& Pampalakis, G. Kallikrein-related peptidases: bridges between immune functions and extracellular matrix degradation. Biol. Chem. 391, 321-331 (2010).

33. Takenaka, M. C., Robson, S. \& Quintana, F. J. Regulation of the T Cell Response by CD39. Trends Immunol. 37, 427-439 (2016).

34. Vijayan, D., Young, A., Teng, M. W. L. \& Smyth, M. J. Targeting immunosuppressive adenosine in cancer. Nat. Rev. Cancer 17, 709 (2017).

35. Smith, G. C. S. First-trimester determination of complications of late pregnancy. JAMA 303, 561$562(2010)$.

36. Pavličev, M. et al. Single-cell transcriptomics of the human placenta: inferring the cell communication network of the maternal-fetal interface. Genome Res. 27, 349-361 (2017).

37. Camp, J. G. et al. Multilineage communication regulates human liver bud development from pluripotency. Nature 546, 533-538 (2017).

38. Puram, S. V. et al. Single-Cell Transcriptomic Analysis of Primary and Metastatic Tumor Ecosystems in Head and Neck Cancer. Cell 171, 1611-1624.e24 (2017). 
39. Skelly, D. A. et al. Single-Cell Transcriptional Profiling Reveals Cellular Diversity and Intercommunication in the Mouse Heart. Cell Rep. 22, 600-610 (2018).

40. Pardoll, D. M. The blockade of immune checkpoints in cancer immunotherapy. Nat. Rev. Cancer 12, 252-264 (2012).

41. Bulmer, J. N., Morrison, L., Longfellow, M., Ritson, A. \& Pace, D. Granulated lymphocytes in human endometrium: histochemical and immunohistochemical studies. Hum. Reprod. 6, 791-798 (1991).

42. King, A., Wellings, V., Gardner, L. \& Loke, Y. W. Immunocytochemical characterization of the unusual large granular lymphocytes in human endometrium throughout the menstrual cycle. Hum. Immunol. 24, 195-205 (1989).

Acknowledgments: We thank G.Graham, J.Shilts, A.Lopez, N.Reuter, S.Orchard and P.Porras for discussions on CellPhoneDB; D.Dixon, D.Popescu, J.Fletcher, O.Chazara, L. Mamanova, A. Jinat, C.I.Mazzeo for experimental help; D.McDonald and D.Bulmer for assistance with flow cytometry experiments and imaging; A.Hupalowska for help on the illustration; S. Lindsay, A. Farnworth, HDBR, P.Ayuk and the Newcastle Uteroplacental Tissue Bank for providing samples; R.Rostom, D.McCarthy, V.Svensson, M.Hemberg and T.Gomes for computational discussions and all members of the Teichmann and Behjati lab for further discussions on the manuscript. We are indebted to the donors for participating in this research. R.V-T is supported by an EMBO Long-Term Fellowship and a Human Frontier Science Program Long-Term Fellowship.

\section{Reviewer information:}

Author contributions: R.V-T and S.A.T conceived the study. Sample/library preparation was performed by R.V-T with contributions from M.Y.T, J.P, E.S. and S.L; FACS experiments were performed by R.V-T, R.A.B, A.F, A.M.S, R.P.P and M.A.I.; Histology staining was performed by J.N.B, L.G, R.V-T, M.Y.T, B.M, B.I., S.H, D.H.R and A.W-C; M.E. and R.V-T analysed and interpreted the data with contributions from M.V-T, M.J.T.S, L.W, G.W, A.G, A.Z, J.H, K.B.N, K.P, M.H, A.M. and S.A.T; R.V-T, A.M, and S.A.T. wrote the manuscript with contributions from M.H., M.E., K.B.M. and M.Y.T; M.H, A.M., and S.A.T. co-directed the study. All authors read and accepted the manuscript.

Competing Interests: The authors declare no competing interests.

Extended data is available for this paper at 
Supplementary information is available for this paper at

Reprints and permissions information is available at www.nature.com/reprints.

Correspondence and requests for materials should be addressed to S.A.T., A.M. or M.H.

Publisher's note: Springer Nature remains neutral with regard to jurisdictional claims in published maps and institutional affiliations.

Fig. 1 | Identification of novel cell types at the maternal-fetal interface. a, Diagram illustrating the decidual-placental interface in early pregnancy. b, Workflow for single cell transcriptome profiling of decidua, placenta and maternal PBMC. c, Placental and decidual cell clusters from 10x and SS2 scRNAseq analysis visualised by UMAP. Colors indicate cell type/state. Decidua $(n=11)$, placenta $(n=5)$ and blood $(\mathrm{n}=6)$. $\mathbf{d}$, UMAP visualisation of $\mathrm{T}$ cell clonal expansion and clusters by integrating SS2 and 10x T cell data on clusters 4, 8, 10, 15 from Fig.1c e, Origin of droplet cells in Fig 1c by tissue (above) or genotype (below). Purple circle $=$ maternal cells in placenta; green circle $=$ fetal cells in decidua .

Fig. 2 Ligand-receptor expression during EVT differentiation. a, Pseudotime ordering of trophoblast cells reveals EVT and SCT pathways. Enriched EPCAM ${ }^{+}$and HLA-G ${ }^{+}$cells on placental and decidual isolates are included. Placenta $(n=5)$ and decidua $(n=11)$. b, Violin plots showing logtransformed normalized expression levels for selected ligand-receptor pairs which change during pseudotime and predicted significant by CellPhoneDB (EGFR, HBEGF, NRP2, PGF, MET, HGF, $A C K R 2$, CCL5, CXCR6, CXCL16, TGFb, TGFBR2, TGFBR1). Cells from Fig. 1c are used for the violin plots.

Fig. 3 Stromal distribution in the two distinct decidual layers. a, Heatmap showing relative expression (z-score) of selected genes for perivascular (PV) and decidual stromal (dS) cells ( $\mathrm{n}=11$; adj $p$-value $<0.1$; Wilcoxon rank-sum test with bonferroni correction) $\mathbf{b}$, IHCs of a spiral artery in serial sections of the decidua stained for CD34 (endothelial cells), ACTA2 (perivascular -PV and decidual stromal 1-dS1), MCAM (PV1) and MMP11 (PV2) (biological n=2). Scale bar $=100 \mu \mathrm{m}$. c, IHC of decidual sections stained for ACTA2 which distinguishes $\mathrm{ACTA}^{+} \mathrm{dS} 1$ in decidua spongiosa vs. ACTA2 $^{-}$dS2/3 in decidua compacta. Right panels are higher magnification of inset. Scale bar $=50 \mu \mathrm{m}$. (biological $\mathrm{n}=3$ ). $\mathbf{d}$, multiplexed smFISH of decidua parietalis showing two decidual layers. ACTA2 ${ }^{+}$ dS1 in decidua spongiosa; $I G B P 1^{+}$and $\mathrm{PRL}^{+} \mathrm{dS} 2 / 3$ confined to decidua compacta (biological $\mathrm{n}=2$ ). e, Heatmap shows selected significant ligand-receptor interactions $(n=6, p$-value $<0.05$, permutation test, see Methods) between PV and dS (left) and decidual cells (right) ( $\mathrm{n}=11)$.

Fig. 4 | Three dNK populations. a, Heatmap showing relative expression (z-score) of markers defining the three dNK subsets $(\mathrm{n}=11$; pct. $1>10 \%$, pct. $2<60 \%$; $p$-value $<0.1$ after bonferroni correction, Wilcoxon rank-sum test). b, KIRid method workflow $\mathbf{c}$, z-scores of KIR receptors (mean expression 
levels). Expression values were generated using SS2 data and the KIRid approach $(n=5)$ d, FACS gating strategy to identify dNK subsets (biological $n=6$; Supplementary table 9). e, z-scores of expression of granule molecules PRF1, GNL1, GZMA, GZMB in dNK subsets, (n=11). f, Flow cytometry to compare

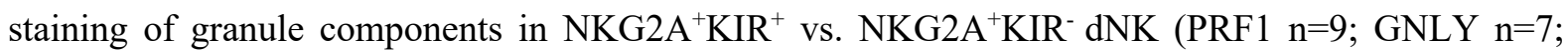
GZMA $n=8$; GZMB $n=10$; Supplementary table 9). Non-parametric paired Wilcoxon test $\left({ }^{*} p<0.05\right.$, $* * p<0.01$ ); $\mathbf{g}$, z-scores of glycolysis enzymes (mean expression), where only differentially expressed enzymes are plotted ( $\mathrm{n}=11 ; p$-value $<0.1$ after bonferroni correction, Wilcoxon rank-sum test).

Fig. 5 | Multiple regulatory immune and inflammatory responses at the site of placentation, a, Overview of selected ligand-receptor interactions, $p$-values indicated by circle size, scale on right (permutation test, see Methods). The means of the average expression level of interacting molecule 1 in cluster 1 and interacting molecule 2 in cluster 2 are indicated by colour. Only droplet data was used $(n=6)$. $\mathbf{b}$, Diagram of the main receptors and ligands expressed on the three dNK subsets that are involved in cellular recruitment or immunomodulation.

\section{Methods}

\section{Patient samples}

All tissue samples used for this study were obtained with written informed consent from all participants in accordance with the guidelines in The Declaration of Helsinki 2000 from multiple centres.

Human embryo, fetal and decidual samples were obtained from the MRC/Wellcome-funded Human Developmental Biology Resource (HDBR ${ }^{43}$, http://www.hdbr.org) with appropriate maternal written consent and approval from the Newcastle and North Tyneside NHS Health Authority Joint Ethics Committee (08/H0906/21+5). HDBR is regulated by the UK Human Tissue Authority (HTA; www.hta.gov.uk) and operates in accordance with the relevant HTA Codes of Practice. Decidual tissue for smFISH (Extended Data Fig. 7c) was also covered by this ethics.

Peripheral blood from woman undergoing elective terminations were under appropriate maternal written consent and approvals from the Newcastle Academic Health Partners (reference NAHPB-093) and HRA NHS Research Ethics committee North-East-Newcastle North Tyneside 1 (REC reference $12 / \mathrm{NE} / 0395)$

Decidual tissue for immunohistochemistry (Fig. 3b-c, Extended Data Fig.7a and Extended Data Fig. 9c-d) were obtained from elective terminations of normal pregnancies at Addenbrooke's Hospital between 6 and 12 weeks gestation, under ethical approval from the Cambridge Local Research Ethics Committee (04/Q0108/23). 
Decidual tissue for smFISH (Fig. 3d, Extended Data Fig. 6b and Extended Data Fig. 7b) were obtained from the Newcastle Uteroplacental Tissue Bank and Ethics numbers are Newcastle and North Tyneside Research Ethics Committee 1 Ref:10/H0906/71 and 16/NE/0167.

\section{Isolation of decidual, placental and blood cells}

Decidual and placental tissue were washed in HAMS F12 medium, macroscopically separated and then washed for at least 10 mins in RPMI or HAMS F12 medium respectively before processing.

Decidual tissues were chopped using scalpels into approximately $0.2 \mathrm{~mm}^{3}$ cubes and enzymatically digested in $15 \mathrm{ml} 0.4 \mathrm{mg} / \mathrm{mL}$ collagenase V (Sigma, C-9263) solution in RPMI 1640 medium (ThermoFisher Scientific, 21875-034)/10\% FCS (Biosfera, FB-1001) at $37^{\circ} \mathrm{C}$ for $45 \mathrm{~min}$. The supernatant was diluted with medium and passed through 100um cell sieve (Corning, 431752) and then 40um cell sieve (Corning, 431750). The flow-through was centrifuged and resuspended in $5 \mathrm{ml}$ of red blood cell lysis buffer (Invitrogen, 00-4300) for 10min.

Each first trimester placenta was placed in a petri dish and the placental villi were scraped from the chorionic membrane using a scalpel. The stripped membrane was discarded and the resultant villous tissue was enzymatically digested in $70 \mathrm{ml} \mathrm{0.2 \%} \mathrm{trypsin} 250$ (Pan Biotech P10-025100P)/0.02\% EDTA (Sigma E9884) in PBS with stirring at $37^{\circ} \mathrm{c}$ for $9 \mathrm{~min}$. The disaggregated cell suspension was passed through sterile muslin gauze (Winware food grade) and washed through with Hams F12 medium (Biosera SM-H0096) containing 20\% FBS (Biosera FB-1001). Cells were pelleted from the filtrate by centrifugation and re-suspended in Hams F12. The undigested, gelatinous tissue remnant was retrieved from the gauze and further digested with $10-15 \mathrm{ml}$ collagenase $\mathrm{V}$ at $1.0 \mathrm{mg} / \mathrm{ml}$ (Sigma C9263) in Hams $\mathrm{F} 12 \mathrm{medium} / 10 \% \mathrm{FBS}$ with gentle shaking at $37^{\circ} \mathrm{C}$ for $10 \mathrm{~min}$. The disaggregated cell suspension from collagenase digestion was passed through sterile muslin gauze and the cells pelleted from the filtrate as before. Cells obtained from both enzyme digests were pooled together and passed through 100um cell sieve (Corning, 431752) and washed in Hams F12. The flow-through was centrifuged and resuspended in $5 \mathrm{ml}$ of red blood cell lysis buffer (Invitrogen, 00-4300) for 10min.

Blood samples were carefully layered onto a Ficoll-paque gradient (Amersham, Buckinghamshire, UK) and centrifuged at 2,000 rpm for $30 \mathrm{~min}$ without breaks. Peripheral blood mononuclear cells (PBMCs), from the interface between the plasma and the Ficoll-Paque gradient, were collected and washed in icecold phosphate-buffered saline (PBS), followed by centrifugation at 2,000 rpm for $5 \mathrm{~min}$. The pellet was resuspended in $5 \mathrm{ml}$ of red blood cell lysis buffer (Invitrogen, 00-4300) for 10min.

\section{Assignment of fetal developmental stage}

Up to 8 post conception weeks (PCW), embryos are staged using the Carnegie staging method ${ }^{44}$. At fetal stages beyond 8 PCW, age was estimated from measurements of foot length and heel to knee length. These were compared with a standard growth chart ${ }^{45}$. 


\section{Flow cytometry staining, cell sorting and single-cell RNA sequencing}

Decidual and blood cells were incubated at $4{ }^{\circ} \mathrm{C}$ with $2.5 \mathrm{ul}$ of antibodies in $1 \% \mathrm{FBS}$ in DPBS without Calcium and Magnesium (ThermoFisher Scientific, 14190136). DAPI was used for live/dead discrimination. We used an antibody panel designed to enrich for certain populations for single-cell sorting and single-cell RNA sequencing (scRNA-seq). Cells were sorted using a Becton Dickinson (BD) FACS Aria Fusion with 5 excitation lasers (355nm, 405nm, 488nm, 561nm and 635nm Red), and 18 fluorescent detectors plus forward and side scatter. The sorter was controlled using BD FACS DIVA software (version 7). The antibodies used are listed in Supplementary Table 10.

For single-cell RNA-seq using the plate-based SS2 protocol, we created overlapping gates that comprehensively and evenly sampled all immune cell population in the decidua (Extended Data Fig 1). $\mathrm{B}$ cells $\left(\mathrm{CD} 19^{+}\right.$or $\left.\mathrm{CD} 20^{+}\right)$were excluded from our analysis, due to their absence in decidua ${ }^{46}$. Single cells were sorted into 96-well full-skirted Eppendorf plates chilled to 4C, prepared with lysis buffer consisting of $10 \mathrm{ul}$ of TCL buffer (Qiagen) supplemented with 1\% b-mercaptoethanol. Single-cell lysates were sealed, vortexed, spun down at $300 \mathrm{~g}$ at $4{ }^{\circ} \mathrm{C}$ for $1 \mathrm{~min}$, immediately placed on dry ice, and transferred for storage at $-80^{\circ} \mathrm{C}$. The SS2 protocol was performed on single cells as described previously $y^{47,48}$, with some modifications ${ }^{49}$. Libraries were sequenced aiming at an average depth of 1 million reads/cell, on an Illumina HiSeq 2000 with v4 chemistry (paired-end 75-bp reads).

For the droplet scRNA-seq methods, blood and decidual cells were sorted into immune $\left(\mathrm{CD} 45^{+}\right)$and non-immune $\left(\mathrm{CD} 45^{-}\right)$fractions. B cells $\left(\mathrm{CD} 19^{+}\right.$or $\left.\mathrm{CD} 20^{+}\right)$were excluded from blood analysis, due to their absence in decidua ${ }^{46}$. Only viable cells were considered. Placental cells were stained for DAPI and only viable cells were sorted. In order to improve trophoblast trajectories, an additional enrichment of $\mathrm{EPCAM}^{+}$and HLA-G ${ }^{+}$was performed for selected samples (Fig. 2 only). Cells were sorted into an Eppendorf tube containing PBS with $0.04 \%$ BSA. Cells were immediately counted using a Neubauer hemocytometer and loaded in the 10x-Genomics Chromium. 10x-Genomics v2 libraries were prepared as per the manufacturer's instructions. Libraries were sequenced aiming at a minimum coverage of 50,000 raw reads per cell on an Illumina HiSeq 4000 (paired-end, Read 1: 26 cycles; i7 index:8 cycles, i5 index: 0 cycles. Read 2: 98 cycles).

\section{Flow cytometry staining for granule proteins}

For intracellular staining of granule proteins, dNK were surface stained for 30 mins in FACS buffer with antibodies (listed in Supplementary Table 10). Cells were washed with FACS buffer followed by staining with dead cell marker (DCM Aqua) and strepavidinQ605. dNK were then treated with FIX \& PERM (Thermofisher) and stained for granule proteins. Samples were run on an LSRFortessa FACS analyser (BD Biosciences) and data analysed using FlowJo (Tree Star). dNK were gated as CD3-CD14$\mathrm{CD} 19-$, live cells then $\mathrm{CD}^{2} 6^{+} \mathrm{NKG} 2 \mathrm{~A}^{+}$and then $\mathrm{KIR}^{+}$and $\mathrm{KIR}^{-}$subsets generated using Boolean 
functions with the gates for all the different KIRs stained (KIR $)$, and its inverse gate (KIR'). Wilcoxon test was used to compare granule protein staining between paired dNK subsets from the same donor. A $\mathrm{p}$ value $<0.05$ was considered statistically significant.

\section{Immunohistochemistry (IHC)}

4um tissue sections from formalin-fixed paraffin wax-embedded human decidual and placental tissueswere dewaxed with Histoclear, cleared in $100 \%$ ethanol and rehydrated through gradients of ethanol to PBS. Sections were blocked with $2 \%$ serum (of species in which the secondary antibody was made) in PBS, incubated with primary antibody for overnight at $4 \mathrm{RT}^{\circ} \mathrm{C}$ and slides washed in PBS. Biotinylated horse anti-mouse or goat anti-rabbit secondary antibody were used, followed by vectastain ABC-HRP reagent (Vector, PK-6100) and developed with di-aminobenzidine (DAB) substrate (Sigma, D4168). Sections were counterstained with Carazzi's haematoxylin and mounted in glycerol/gelatin mounting medium (Sigma, GG1-10). Primary antibody was replaced with equivalent concentrations of mouse or rabbit IgG for negative controls. See Supplementary Table 10 for antibody information. Tissue sections were imaged using a Zeiss Axiovert Z1 microscope and Axiovision imaging software SE64 V4.8.

\section{SmFISH}

Samples were fixed in $10 \% \mathrm{NBF}$, dehydrated through an ethanol series and embedded in paraffin wax. $5 \mathrm{mM}$ were cut, baked at $60^{\circ} \mathrm{C}$ for 1 hour and processed using standard pre-treatment conditions, as per the RNAScope multiplex fluorescent reagent kit v2 assay protocol (manual) or the RNAScope 2.5 LS fluorescent multiplex assay (automated). TSA-plus fluorescein, Cy3 and Cy5 fluorophores were used at 1:1500 dilution for the manual assay or 1:300 dilution for the automated assay. Slides were imaged on different microscopes: Hamamatsu Nanozoomer S60 (Extended figure 7c). Zeiss Cell Discoverer 7 (Figure 4d, Extended Figure 7c, Extended Figure 6). Filter details: DAPI: Ex 370-400, BS 394, Em 460-500; FITC: Ex 450-488, BS 490, Em 500-55; Cy3: Ex 540-570, BS 573, Em 540-570; Cy5: Ex 615-648, BS 691, Em 662-756. Camera Hamamatsu ORCA-Flash4.0 V3 sCMOS camera.

\section{Whole genome sequencing}

Tissue DNA and RNA were extracted from fresh frozen samples using the AllPrep DNA/RNA/miRNA kit (Qiagen) following the manufacturer's instructions. Short insert (500bp) genomic libraries were constructed, flowcells prepared and 150 base pair paired-end sequencing clusters generated on the Illumina HiSeq X platform according to Illumina no-PCR library protocols to an average of $30 \times$ coverage. Genotype information is provided in Supplementary Table 1. 


\section{Single cell RNA-seq data analysis}

Droplet-based sequencing data was aligned and quantified using the Cell Ranger Single-Cell Software Suite (version 2.0, 10x Genomics Inc) ${ }^{50}$ against the GRCh38 human reference genome provided by Cell Ranger. Cells with fewer than 500 detected genes and for which the total mitochondrial gene expression exceeded 20\% were removed. Genes that were expressed in fewer than 3 cells were also removed.

SmartSeq2 sequencing data was aligned with HISAT2 ${ }^{51}$, using the same genome reference and annotation as the 10x data. Gene-specific read counts were calculated using HTSeq-count ${ }^{52}$. Cells with fewer than 1,000 detected genes and more than 20\% mitochondrial gene expression content were removed. Further, mitochondrial genes and genes expressed in fewer than 3 cells were also removed. To remove batch effects due to background contamination of cell free RNA, we also removed a set of genes that had a tendency to be expressed in ambient RNA (PAEP, HBG1, HBA1, HBA2, HBM, AHSP, $H B G 2)$.

Downstream analysis such as normalisation, SNN graph-based clustering, differential expression analysis and visualisation, were performed using the R package Seurat ${ }^{53}$ (version 2.3.3). Droplet-based and SmartSeq2 data were integrated using Canonical Correlation Analysis (CCA) implemented in the Seurat alignment workflow ${ }^{54}$. Cells whose expression profile could not be well-explained by lowdimensional CCA, compared to low-dimensional PCA were discarded, as recommended by the Seurat alignment tutorial. Clusters were identified using the community identification algorithm as implemented in the Seurat "FindClusters" function. The SNN graph was constructed using between 5 and 40 canonical correlation vectors as determined by the dataset variability; the resolution parameter to find the resulting number of clusters was tuned so that it produced a number of clusters large enough to capture most of the biological variability. UMAP analysis was performed using the RunUMAP function with default parameters. Differential expression analysis was performed based on the Wilcoxon rank-sum test. The $p$-values were adjusted for multiple testing using the bonferroni correction. Clusters were annotated using canonical cell type markers. Two clusters of peripheral blood monocytes represented the same cell type and were therefore merged.

We further remove contaminating cells: i) maternal stromal cells that were gathered in the placenta for one of the fetuses; ii) a shared decidual/placental cluster with fetal cells mainly present in two fetuses which we think is likely to be contaminating cells from other fetal tissues due to the surgical procedure. This can occur due to the source of the tissue, and the trauma of surgery. We also removed a cluster for which the top markers were genes associated with dissociation-induced effects ${ }^{55}$. Each of the remaining clusters contained cells from multiple different fetuses, indicating that the cell types and states we observed are not impacted by batch effects.

We found further diversity within the T cell clusters, as well as the clusters of endothelial, epithelial and perivascular cells, which we then reanalysed and partitioned separately, using the same alignment and clustering procedure. 
The trophoblasts clusters (clusters 1, 9, 20,13 and 16 from Fig 1.d) were taken from the initial analysis of all cells and merged with the enriched EPCAM+ and HLA-G+ cells. The droplet-based and SS2 datasets were integrated and clustered using the same workflow as described above. Only cells that were identified as trophoblasts were considered for trajectory analysis.

Trajectory modelling and pseudotemporal ordering of cells was performed with the monocle $2 \mathrm{R}$ package $^{56}$ (version 2.8.0). The most highly variable genes were used for ordering the cells. To account for the cell cycle heterogeneity in the trophoblast subpopulations, we performed hierarchical clustering of the highly variable genes and removed the set of genes clustering with known cell cycle genes such as CDK1. Genes which changed along the identified trajectory were identified by performing a likelihood ratio test using the function differentialGeneTest in the monocle 2 package.

Network visualisation was done using Cytoscape (version 3.5.1). The decidual network was created considering only edges with more than 30 interactions. The networks layout was set to force-directed layout.

\section{KIR typing}

Polymerase chain reaction-sequence-specific primer (PCR-SSP) was performed to amplify the genomic DNA for presence or absence of $12 \mathrm{KIR}$ genes $(2 D L 1,2 D L 2,2 D L 3,2 D L 5,3 D L 1,2 D S 1,2 D S 2,2 D S 3$, $2 D S 4,2 D S 5$, and $3 D S 1$ ) and the pseudogene 2DP1. KIR2DS4 alleles were also typed as being either full length or having the 22-bp deletion that prevents cell surface expression. Two pairs of primers were used for each gene, selected to give relatively short amplicons of $100-800 \mathrm{bp}$, as previously described ${ }^{57}$. Extra KIR primers were designed using sequence information from the IPD-KIR database (release 2.4.0) to detect rare alleles of KIR2DS5 and KIR2DL3 (KIR2DS5, 2DS5rev2: TCC AGA GGG TCA CTG GGA and KIR2DL3, 2DL3rev3: AGA CTC TTG GTC CAT TAC CG) ${ }^{58}$. KIR haplotypes were defined by matrix subtraction of gene copy numbers using previously characterized common and contracted KIR haplotypes using the KIR Haplotype Identifier software (www.bioinformatics.cimr.cam.ac.uk/haplotypes).

\section{Inferring maternal/fetal origin of single cells from droplet-based scRNA-seq using whole-genome sequencing variant calls}

To match the processing of the whole-genome sequencing datasets, droplet-based sequencing data from decidua and placenta samples were realigned and quantified against the GRCh37 human reference genome using the Cell Ranger Single-Cell Software Suite (v.2.0 $)^{50}$. The fetal or maternal origin of each barcoded cell was then determined using the tool demuxlet ${ }^{59}$. Briefly, demuxlet can be used to deconvolve droplet-based scRNA-seq experiments in which cells are pooled from multiple, genetically distinct individuals. Given a set of genotypes corresponding to these individuals, demuxlet infers the most likely genetic identity of each droplet by estimating the likelihood of observing scRNA-seq reads 
from the droplet overlapping known SNPs. Demuxlet inferred the identities of cells in this study by analyzing each Cell Ranger-aligned BAM file from decidua and placenta in conjunction with a VCF containing the high-quality WGS variant calls from the corresponding mother and fetus. Each droplet was assigned to be maternal, fetal, or unknown in origin (ambiguous or potential doublet), and these identities were then linked with the transcriptome-based cell clustering data to confirm the maternal and fetal identity of each annotated cell type.

\section{TCR analysis by TraCeR}

The TCR sequences for each single $\mathrm{T}$ cell were assembled using $\mathrm{TraCe}^{60}$ which allowed the reconstruction of the TCRs from scRNA-seq data and their expression abundance (transcripts per million, TPM), as well as identification of the size, diversity and lineage relation of clonal subpopulations. In total, we obtained the TCR sequences for 1,482 $\mathrm{T}$ cells with at least one paired productive $\alpha \beta$ or gamma-delta chain. Cells for which more than two recombinants were identified for a particular locus were excluded from further analysis.

\section{Whole-genome sequencing alignment and variant calling}

Maternal and fetal whole-genome sequencing data were mapped to the GRCh37.p13 reference genome using BWA-MEM v.0.7.15 ${ }^{61}$. The SAMtools ${ }^{62}$ fixmate utility (v.1.5) was used to update read pairing information and mate-related flags. Reads near known indels from the Mills ${ }^{63}$ and $1000 \mathrm{G}^{64}$ gold standard reference set for hg19/GRCh37 were locally realigned using GATK IndelRealigner v.3.763. Base calling assessment and base quality scores were adjusted with GATK BaseRecalibrator and PrintReads v.3.7 $7^{62,65}$. PCR duplicates were identified and removed using Picard MarkDuplicates v.2.14.1 $1^{65,66}$. Finally, bcftools mpileup and call v.1. $6^{67}$ were used to produce genotype likelihoods and output called variants at all known biallelic SNP sites overlapping protein-coding genes. For each sample, variants called with phred-scale quality score (QUAL) $>=200$, at least 20 supporting reads (DP $>=20$ ), and mapping quality $(\mathrm{MQ})>=60$, were retained as high-quality variants.

\section{Quantification of KIR gene expression by KIRid}

The KIR locus is highly polymorphic both in terms of numbers of genes and alleles ${ }^{68}$. Including a single reference sequence for each gene can lead to reference bias for donors that happen to match the reference sequence better. To address these issues, we used a tailored approach in which we first built a total cDNA reference by concatenating the Ensembl coding and non-coding transcript sequences, excluding transcripts belonging to the KIR genes (GRCh38, v.90), and the full set of known KIR cDNAs sequences from the IPD-KIR database ${ }^{69}$ (release 2.7.0). For each donor, we removed transcript sequences for KIR genes determined to be absent in that individual, which decreases the extent of multi- 
mapping/quantification. Each donor's single cell reads were then mapped to the corresponding donorspecific reference using Kallisto ${ }^{70}$ (v0.43.0 with default options). Expression levels were quantified using the multi-mapping deconvolution tool $\mathrm{MMSEQ}^{71}$, and gene level estimates were obtained by aggregating over different alleles for each KIR gene.

\section{Cell-cell communication analysis}

To enable a systematic analysis of cell-cell communication molecules, we developed CellPhoneDB, a public repository of ligands, receptors and their interactions. Our repository relies on the use of public resources to annotate receptors and ligands. We include subunit architecture for both ligands and receptors, in order to accurately represent heteromeric complexes.

Ligand-receptor pairs are defined based on physical protein-protein interactions (PPI) (see CellPhoneDB annotation). We provide CellPhoneDB with a user-friendly web interface at CellPhoneDB.org, where the user can search for ligand-receptor complexes and interrogate their own single-cell transcriptomics data.

To assess cellular crosstalk between different cell types, we used our repository in a statistical framework for inferring cell-cell communication networks from single cell transcriptome data. We derived enriched receptor-ligand interactions between two cell types based on expression of a receptor by one cell type and a ligand by another cell type, using the droplet-based data. In order to identify the most relevant interactions between cell types, we looked for the cell-type specific interactions between ligands and receptors. Only receptors and ligands expressed in more than $10 \%$ of the cells in the specific cluster were considered.

We performed pairwise comparisons between all cell types. First, we randomly permuted the cluster labels of all cells 1000 times and determined the mean of the average receptor expression level of a cluster and the average ligand expression level of the interacting cluster. For each receptor-ligand pair in each pairwise comparison between two cell types, this generated a null distribution. By calculating the proportion of the means which are "as or more extreme" than the actual mean, we obtained a $p$ value for the likelihood of cell type-specificity of a given receptor-ligand complex. We then prioritized interactions that are highly enriched between cell types based on the number of significant pairs and manually selected biologically relevant ones. For the multi-subunit heteromeric complexes, we required that all subunits of the complex are expressed (using a threshold of $10 \%$ ), and therefore we used the member of the complex with the minimum average expression to perform the random shuffling.

\section{CellPhoneDB annotation: Membrane, secreted and peripheral proteins}

Secreted proteins were downloaded from Uniprot using KW-0964 (secreted). Secreted proteins were annotated as cytokines (KW-0202), hormones (KW-0372), growth factors (KW-0339) and immune- 
related using Uniprot keywords and manual annotation. Cytokines, hormones, growth factors and other immune related proteins were annotated as "secreted highlight" proteins in our lists.

Plasma membrane proteins were downloaded from Uniprot using KW-1003 (cell membrane). Peripheral proteins from the plasma membrane were annotated using the Uniprot Keyword SL-9903, and the remaining proteins were annotated as transmembrane proteins. We completed our lists of plasma transmembrane proteins by doing an extensive manual curation by literature mining and Uniprot description of proteins with transmembrane and immunoglobulin-like domains.

Plasma membrane proteins were annotated as receptors and transporters. Transporters were defined by the Uniprot keyword KW-0813. Receptors were defined by the Uniprot keyword KW-0675. The list of receptors was extensively reviewed and new receptors were added based on Uniprot description and bibliography revision. Receptors involved in immune cell communication were carefully annotated.

Protein lists are available at https://www.cellphonedb.org/downloads. Three columns indicate whether the protein has been manually curated: "tags", "tags_description", "tags_reason".

"tags" is related to the manual curation of a protein. Three options: i) N/A: the protein has not been manually curated; ii) To_add: Secreted and/or plasma membrane protein annotation is added; iii) To_comment: the protein is either secreted (KW-0964) or membrane-associated (KW-1003), but we manually added a specific property of the protein (i.e. the protein is annotated as a receptor)

"tags_reason" is related to the protein properties. Five options: i) "extracellular_add": the protein is manually annotated as plasma membrane; ii) "peripheral_add": the protein is manually annotated as peripheral protein instead of plasma membrane; iii) "secreted_add": the protein is manually annotated as secreted; iv) "secreted_high": the protein is manually annotated as secreted highlight. Cytokines, hormones, growth factors and other immune-related proteins; v) "receptor_add": the protein is manually annotated as a receptor.

"tags_description" is a brief description of the protein, function or property related to the manually curated protein.

\section{CellPhoneDB annotation: heteromeric receptors and ligands}

Heteromeric receptors and ligands (i.e. proteins that are complexes of multiple gene products) were annotated by reviewing the literature and Uniprot descriptions. Cytokine complexes, TGF family complexes and integrin complexes were carefully annotated.

If heteromers are defined in PDB (http://www.rcsb.org/), structural information is included in our CellPhoneDB annotation. Heteromeric complex lists are available at CellPhoneDB.org.

\section{CellPhoneDB annotation: Interactions}

The majority of ligand-receptor interactions were manually curated by reviewing Uniprot descriptions and PubMed information on membrane receptors. Cytokine and chemokine interactions are annotated following the "International Union of Pharmacology annotation" ${ }^{72}$. Other groups of cell surface proteins 
whose interactions were manually reviewed include the TGF family, integrins, lymphocyte receptors, semaphorins, ephrins, notch and TNF receptors.

In addition, we considered interacting partners as:

i) Binary interactions annotated by IUPHAR (http://www.guidetopharmacology.org/)

ii) Cytokines, hormones and growth factors interacting with receptors annotated by the iMEX consortium (https://www.imexconsortium.org/) ${ }^{73}$

We excluded from our analysis transporters and a curated list of proteins including: i) Co-receptors; ii) Nerve-specific receptor like those related to ear-binding, olfactory receptor, taste receptor and salivary, iii) Small molecule receptors, iv) Immunoglobulin chains; v) Pseudogenes; vi) Viral and retroviral proteins, pseudogene, cancer antigens and photoreceptors. These proteins are annotated as "others" in the "protein list". We also excluded from our analysis a list of interacting partners not directly involved in cell-cell communication. The "remove interactions" list is available in https://www.cellphonedb.org/downloads .

Lists of interacting protein chains are available in https://www.cellphonedb.org/downloads. The column "source" indicates the curation source. Manually curated interactions are annotated as "curated", and bibliography used to annotate the interaction is stored in "comments_interaction". "Uniprot" means that the interaction has been annotated using UniProt descriptions.

\section{Linking Ensembl and Uniprot id}

We assigned to the custom curated interaction list all the Ensembl gene id's by matching information from Uniprot and Ensembl by the gene name.

\section{Database structure}

Information is stored in a PostgreSQL relational database (www.postgresql.org). SQLAlchemy (www.sqlalchemy.org) and Python 3 was used to build the database structure and the query logic. All the code is open source and uploaded in the webserver.

\section{Data and materials availability}

Our expression data for different tissues is also available for user-friendly interactive browsing online at http://data.teichlab.org (Maternal-Fetal Interface). The raw sequencing data, expression count data with cell classifications and the WGS data are deposited at ArrayExpress: experiments: E-MTAB-6701 (for droplet-based data), E-MTAB-6678 (for SS2 data), E-MTAB-7304 (for the WGS data). Our CellPhoneDB repository is available in cellphonedb.org. 


\section{Code availability}

CellPhoneDB code is available in github.org/Teichlab/. The code can be downloaded also from: https://cellphonedb.org/downloads.

KIRid can be downloaded from https://github.com/Teichlab/KIRid

43. Gerrelli, D., Lisgo, S., Copp, A. J. \& Lindsay, S. Enabling research with human embryonic and fetal tissue resources. Development 142, 3073-3076 (2015).

44. Smith, R. J. H. Human embryology and teratology. By Roana O'Rahilla, Fabiola Muller. WileyLiss, Inc., New York, 1992, 336 pp, \$159.95. Head Neck 15, 586-586 (1993).

45. Warren, M. \& Hern, M. D. Correlation of fetal age and measurements between 10 and 26 weeks of gestation. Obstet. Gynecol. 63, (1984).

46. Bulmer, J. N., Williams, P. J. \& Lash, G. E. Immune cells in the placental bed. Int. J. Dev. Biol. 54, 281-294 (2010).

47. Picelli, S. et al. Full-length RNA-seq from single cells using Smart-seq2. Nat. Protoc. 9, 171$181(2014)$.

48. Trombetta, J. J. et al. Preparation of Single-Cell RNA-Seq Libraries for Next Generation Sequencing. in Current Protocols in Molecular Biology 4.22.1-4.22.17 (2014).

49. Villani, A.-C. et al. Single-cell RNA-seq reveals new types of human blood dendritic cells, monocytes, and progenitors. Science 356, (2017).

50. Zheng, G. X. Y. et al. Massively parallel digital transcriptional profiling of single cells. Nat. Commun. 8, 14049 (2017).

51. Kim, D., Langmead, B. \& Salzberg, S. L. HISAT: a fast spliced aligner with low memory requirements. Nat. Methods 12, 357-360 (2015).

52. Anders, S., Pyl, P. T. \& Huber, W. HTSeq--a Python framework to work with high-throughput sequencing data. Bioinformatics 31, 166-169 (2015).

53. Satija, R., Farrell, J. A., Gennert, D., Schier, A. F. \& Regev, A. Spatial reconstruction of singlecell gene expression data. Nat. Biotechnol. 33, 495-502 (2015). 
54. Butler, A. \& Satija, R. Integrated analysis of single cell transcriptomic data across conditions, technologies, and species. bioRxiv 164889 (2017). doi:10.1101/164889

55. van den Brink, S. C. et al. Single-cell sequencing reveals dissociation-induced gene expression in tissue subpopulations. Nat. Methods 14, 935 (2017).

56. Qiu, X. et al. Reversed graph embedding resolves complex single-cell trajectories. Nat. Methods 14, 979-982 (2017).

57. Hiby, S. E. et al. Combinations of Maternal KIR and Fetal HLA-C Genes Influence the Risk of Preeclampsia and Reproductive Success. J. Exp. Med. 200, 957-965 (2004).

58. Robinson, J. et al. The IPD and IMGT/HLA database: allele variant databases. Nucleic Acids Res. 43, D423-D431 (2014).

59. Kang, H. M. et al. Multiplexed droplet single-cell RNA-sequencing using natural genetic variation. Nat. Biotechnol. 36, 89-94 (2018).

60. Stubbington, M. J. T. et al. T cell fate and clonality inference from single-cell transcriptomes. Nat. Methods 13, 329-332 (2016).

61. Li, H. Aligning sequence reads, clone sequences and assembly contigs with BWA-MEM. arXiv [q-bio.GN] (2013).

62. Li, H. et al. The Sequence Alignment/Map format and SAMtools. Bioinformatics 25, 2078-2079 (2009).

63. Mills, R. E. et al. Natural genetic variation caused by small insertions and deletions in the human genome. Genome Res. 21, 830-839 (2011).

64. 1000 Genomes Project Consortium et al. A map of human genome variation from populationscale sequencing. Nature 467, 1061-1073 (2010).

65. Van der Auwera, G. A. et al. From FastQ data to high confidence variant calls: the Genome Analysis Toolkit best practices pipeline. Curr. Protoc. Bioinformatics 43, 11.10.1-33 (2013).

66. Broad Institute. Picard Tools. Broad Institute, GitHub repository

67. Li, H. A statistical framework for SNP calling, mutation discovery, association mapping and population genetical parameter estimation from sequencing data. Bioinformatics 27, 2987-2993 (2011).

68. Parham, P. \& Moffett, A. Variable NK cell receptors and their MHC class I ligands in immunity, 
reproduction and human evolution. Nat. Rev. Immunol. 13, 133-144 (2013).

69. Robinson, J., Mistry, K., McWilliam, H., Lopez, R. \& Marsh, S. G. E. IPD--the Immuno Polymorphism Database. Nucleic Acids Res. 38, D863-9 (2010).

70. Bray, N. L., Pimentel, H., Melsted, P. \& Pachter, L. Near-optimal probabilistic RNA-seq quantification. Nat. Biotechnol. 34, 525 (2016).

71. Turro, E. et al. Haplotype and isoform specific expression estimation using multi-mapping RNAseq reads. Genome Biol. 12, R13 (2011).

72. Bachelerie, F. et al. International Union of Basic and Clinical Pharmacology. [corrected]. LXXXIX. Update on the extended family of chemokine receptors and introducing a new nomenclature for atypical chemokine receptors. Pharmacol. Rev. 66, 1-79 (2014).

73. Orchard, S. et al. Protein interaction data curation: the International Molecular Exchange (IMEx) consortium. Nat. Methods 9, 345-350 (2012).

Extended Data Fig. 1 | Gating strategy for SS2 data. a, Gating strategy for a panel of 14 antibodies to analyse immune cells in decidual samples by SS2 (CD3, CD4, CD8, CD9, CD14, CD16, CD19, CD20, CD34, CD45, CD56, CD94, DAPI, HLA-DR, HLA-G). Cells isolated for SS2 data were gated on: live; CD19/20-ve, singlets, and the following cell types sorted: i) $\mathrm{CD}^{+} 5^{+}, \mathrm{CD}^{4}{ }^{++}, \mathrm{HLA}^{-\mathrm{DR}}{ }^{++}$; ii)

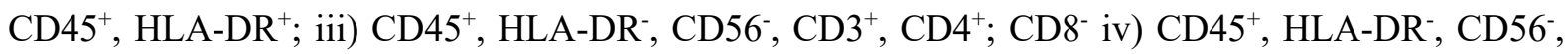

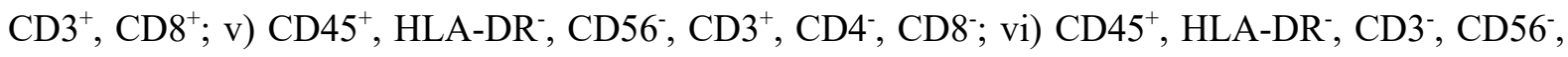
CD94- (all -); vii) CD45 ${ }^{+}$, HLA-DR', CD3-, CD56 ${ }^{+}, \mathrm{CD} 94-$; viii) autofluorescence; ix) CD45+, HLADR-, CD3-, CD56+, CD94+, CD9-; x) CD45+, HLA-DR-, CD3-, CD56+, CD94+, CD9+; xi) CD45-, HLA-G+; xii) CD45-, HLA-G-. Sample F9 is shown as an example. Cells from different gates were sorted in different plates: T cells (gates i and ii); Myeloid (gates iii, iv, v) ; NK cells (gates vi, vii, viii, ix, x) ; CD45- (gates xi, xii). Antibody information in Supplementary Table 10.

Extended Data Fig. 2 | Quality control of droplet \& SS2 datasets. a, Histograms show distribution of the cells from the SS2 dataset ordered by number of detected genes and mitochondrial gene expression content; $\mathbf{b}$, Histograms show distribution of the cells from the droplet-based dataset ordered by number of detected genes and mitochondrial gene expression content; $\mathbf{c}$, Total numbers of cells that passed the quality control, processed by SS2 and droplet scRNA-seq. Each row is a separate donor. d, $\mathrm{CC} 1$ and $\mathrm{CC} 2$ canonical correlation vectors of integrated analysis of decidual and placental cells from 
the SS2 (decidua $\mathrm{n}=5$, PBMC $\mathrm{n}=2$ ) and droplet-based datasets (placenta $\mathrm{n}=5$, decidua $\mathrm{n}=6$, blood $\mathrm{n}=4$ ), colored based on their assignment to clusters shows clear separation between the immune; decidual stromal and perivascular cells; fetal fibroblasts and trophoblast populations;

Extended Data Fig. 3 | Overview of droplet \& SS2 datasets. a, UMAP plot showing the integration of the SS2 and droplet-based dataset and the log-transformed expression of MKI67 (marking proliferating cells). b, UMAP plots showing the separate more detailed integration analysis of the cells from cluster 14 (perivascular cells), cluster 19 (endothelial cells) and cluster 25 (epithelial cells). Clusters are labelled as in Fig. 1c. c, UMAP visualisation of T cell clusters obtained by integrating SS2 and droplets $\mathrm{T}$ cells subpopulations $(4,8,10,15$ clusters) from Fig.1c. Cells are colored by the tissue of origin (upper panel) and the identified clusters (lower panel). d, Heatmap showing the z-score of the mean log-transformed normalised counts for each cluster of selected marker genes used to annotate clusters. For a more extensive set of genes see Supplementary Table 2 (adj $p$-value $<0.1$; Wilcoxon ranksum test with bonferroni correction). Acronyms: NK- NK cells; NKp - proliferating NK cells; ILCInnate lymphocyte cells; M- macrophages; HB- Hofbauer cells; MO- monocytes; DC- dendritic cells; Granulo- granulocytes; $\mathrm{CD} 4^{-} \mathrm{CD} 4^{+} \mathrm{T}$ cells; $\mathrm{CD} 8^{-} \mathrm{CD} 8^{+} \mathrm{T}$ cells; MAIT- Mucosal associated invariant T cells; GD- gamma-delta T cells; CD8c- cytotoxic CD8 ${ }^{+} \mathrm{T}$ cells; Plasma- plasma cells; Endo- endothelial cells; Endo f - Fetal endothelial; Endo m - Maternal endothelial; Endo L- Lymphatic endothelial cells; Epi- epithelial cells; PV- perivascular cells; dS- decidual stromal cells; F- fibroblast; VCTcytotrophoblast cells; VCTp- proliferative cytotrophoblast cells SCT- syncytiotrophoblast; EVTextravillous trophoblast; EVTp - proliferative extravillous trophoblast. e, Log-likelihood differences between assignment to fetal vs. assignment to maternal origin of cells based on SNP calling from the droplet RNA-seq data. Cells are colored by their assignment as determined by Demuxlet. For this figure we used placenta $n=5$, decidua $n=6$, blood $n=4$. f, UMAP visualisation of the log-transformed normalised expression of selected marker genes of the M3 subpopulation.

\section{Extended Data Fig. 4 | Cell-cell communication networks in the maternal-fetal interface using} CellPhoneDB. a, Information aggregated within CellPhoneDB.org b, Statistical framework to infer ligand-receptor complex specific to two cell types from single cell transcriptomics data. Predicted $p$ values for ligand-receptor complex across two cell clusters are calculated using permutations, where cells are randomly re-assigned to clusters (see Methods) c, Networks visualising potential specific interactions in the decidua, where nodes are clusters (cell types) and edges represent the number of significant ligand-receptor pairs. The network was created for edges with more than 30 interactions and the network layouts were set to force-directed layout. Only droplet data was considered for the CellPhoneDB analysis $(n=6) \mathbf{d}$, Networks visualising potential specific interactions in the placenta, where nodes are clusters and edges represent the number of significant ligand-receptor pairs. The network layouts were set to force-directed layout. Only droplet data was considered for the analysis (n $=5)$ e, An example of significant interactions identified by CellPhoneDB. Violin plots show log- 
transformed normalized expression levels of the components of the IL6/IL6R complex in placental cells. IL6 expression is enriched in Fibroblast 2 cluster (dark brown, in panel d) and the two subunits of the IL6 receptors (IL6R and IL6ST) are co-expressed in Hofbauer cells.

Extended Data Fig. 5 | Trophoblast analysis. a, UMAP visualisation of the integrated analysis of the trophoblast subpopulations that were used for pseudotime analysis, including the enriched $\mathrm{EPCAM}^{+}$ and HLA-G ${ }^{+}$cells (see Methods). Cells that were excluded from the pseudotime analysis are colored in grey (placenta $n=5$, decidua $n=11$ ). b, UMAP visualisation of the log-transformed normalised expression of selected canonical trophoblast marker genes $(\mathrm{n}=5)$. $\mathbf{c}$, Visualisation of log-transformed normalised expression of $H L A-G, M K I 67, L G A L S 13$ across trophoblast differentiation. d, Heatmap showing genes involved in the EMT transition, identified as varying significantly as EVT differentiate ( $q$-value $<0.1$, likelihood ratio test, $p$-values were adjusted for the False Discovery Rate).

Extended Data Fig. 6 | Steroid synthesis. a, Heatmap showing relative expression of enzymes involved in cholesterol and steroid synthesis in the three stromal subsets $(n=11)$. $\mathbf{b}$, multiplexed smFISH in two decidua parietalis sections from two different individuals showing an enrichment of CYP11A1 expression in the decidua compacta. Section stained by CYP11A1; LDLR and DAPI. 40x magnification. High resolution is needed to detect differences between the sections. $(n=2)$.

Extended Data Fig. 7 | In situ staining for the different stromal cells. a, IHC of decidual serial sections stained for cytokeratin (uterine glands), CD34 (endothelial cells), ACTA2 (perivascular populations and decidual stromal 1) and IGFBP1 (biological $n=2$ ). ACTA2 ${ }^{+}$stromal cells are confined to the stromal cells of the deeper decidua spongiosa whilst those in the decidua compacta are ACTA2IGFBP $^{+}$stromal cells are enriched in the decidua compacta, whilst those stromal cells around the glands in the decidua spongiosa are IGFBP1 ${ }^{-}$. Glandular secretions are IGFBP1 ${ }^{+}$. Acronyms: Epi $=$ Epithelial glandular cells; $\mathrm{dS}=$ Decidual stromal cells. $\mathbf{b}$, multiplexed smFISH for a decidua parietalis section showing the two decidual layers. $A C T A 2=$ decidual stromal $1(\mathrm{dS} 1)$ population, confined to decidua spongiosa; IGBP1 and $\mathrm{PRL}=$ decidual stromal $3(\mathrm{dS} 2$ and $\mathrm{dS} 3)$ populations confined to decidua compacta. Samples from a different individual than Fig. 4d. Different individual from Fig. 4d (biological $\mathrm{n}=2$ ). $\mathbf{c}$, multiplexed smFISH for a decidua parietalis section showing the two decidual layers. $D K K 1=$ decidual stromal marker; $A C T A 2=$ decidual stromal $1(\mathrm{dS} 1)$ population, confined to decidua spongiosa; $P R L=$ decidual stromal $3(\mathrm{dS} 3)$ population confined to decidua compacta $(\mathrm{n}=1)$.

Extended Data Fig 8 | Lymphocyte populations in the decidua. a, Heatmap showing z-scores of the mean log-transformed normalised expression of selected genes in the lymphocyte populations. Proliferating $\mathrm{dNK}(\mathrm{dNK})$ are excluded from the analysis $(\mathrm{n}=11)$. b, FACS gating strategy in Figure 5 applied in matched blood. Matched blood for sample shown in Figure $5(n=2)$. c, Morphology of 
dNK1, dNK2 and dNK3 by Giemsa-Wright stain after cytospin, Scale bar, $10 \mu \mathrm{m}$ (biological $\mathrm{n}=2$, representative data from $n=1$ shown).

Extended Data Fig 9 | Expression of ligands and receptors at the maternal-fetal interface. a, Heatmap showing z-scores of the mean log-transformed normalised expression of genes annotated as cytokines, growth factors, hormones and angiogenic factors with a $\log$ mean $>0.1$ in the selected decidual immune populations $(\mathrm{n}=11)$. $\mathbf{b}$, Violin plots showing log-transformed normalized expression levels of selected ligands expressed in the three dNK cells and their corresponding receptors expressed on other decidual cells and EVTs (CD39, CD73, ADORA3, CSF1, CSF1R, CCL5, CCR1, XCL1, XCR1; $\mathrm{n}=11) \mathbf{c}$, IHC images of serial decidual sections stained for the EVT marker, HLA-G, and the inhibitory ligand PDL-1. Lower panels are areas in white boxes at higher power. HLA-G ${ }^{+}$cells are only present at the site of placentation (decidua basalis) and are absent elsewhere (decidua parietalis). SpA identifies spiral arteries. The EVT is strongly $\operatorname{PDL}^{+}$(biological $n=5$, we show representative data from one individual). d, IHC images of decidual serial sections of the decidual implantation site (10 week's gestation), stained for the trophoblast cell marker, Cytokeratin-7 (red arrow) and the inhibitory receptor, KIR2DL1 on an NK cell (black arrow). The asterisk (*) marks the lumen of a spiral artery that supplies the conceptus $(\mathrm{n}=5)$.

Extended Data Fig 10 | Encyclopaedia of cells at the maternal-fetal interface. a, Summary of populations from our scRNAseq data. Blue $=$ Fetal, Red $=$ Maternal . 


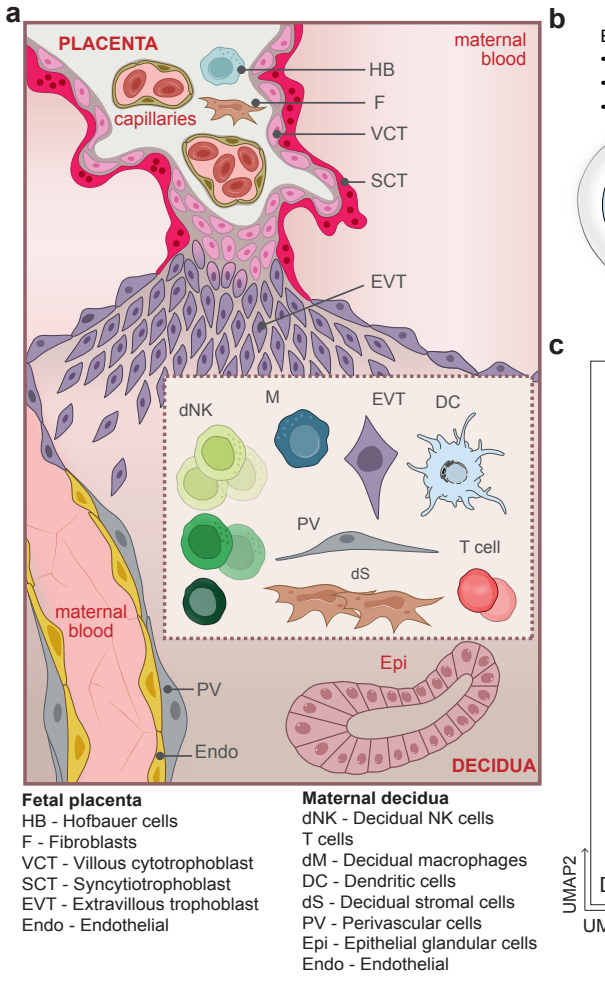
C
Tissue

Cell staining - blood (6)
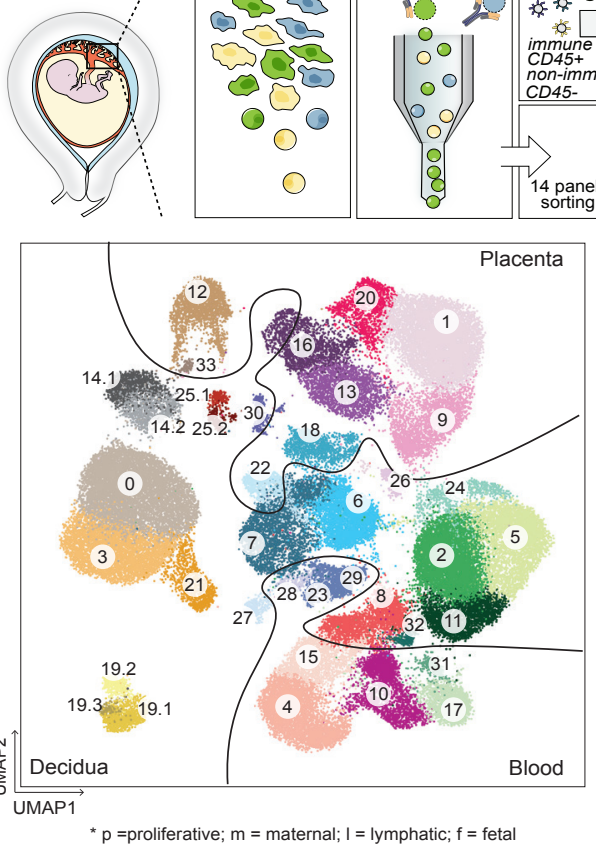

ingle cell RNA-Seq

d
TCR profiling Cl. 4, 8, 10, 15

Blood

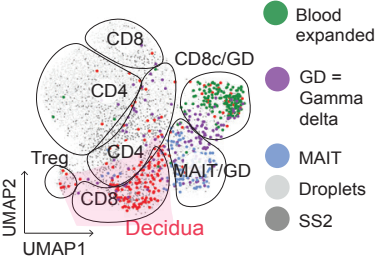

e

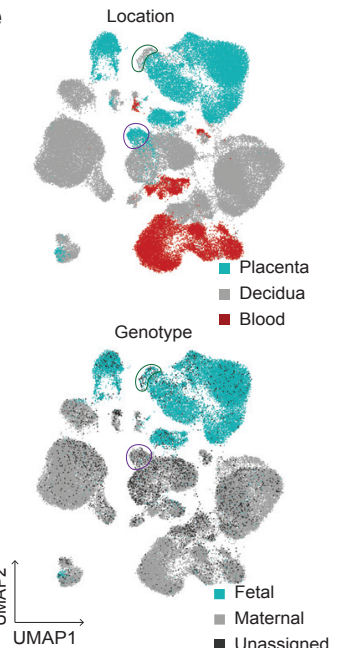




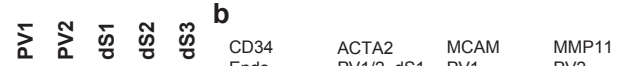

ACTA2, PRL.

ACTA2

|GFBP1
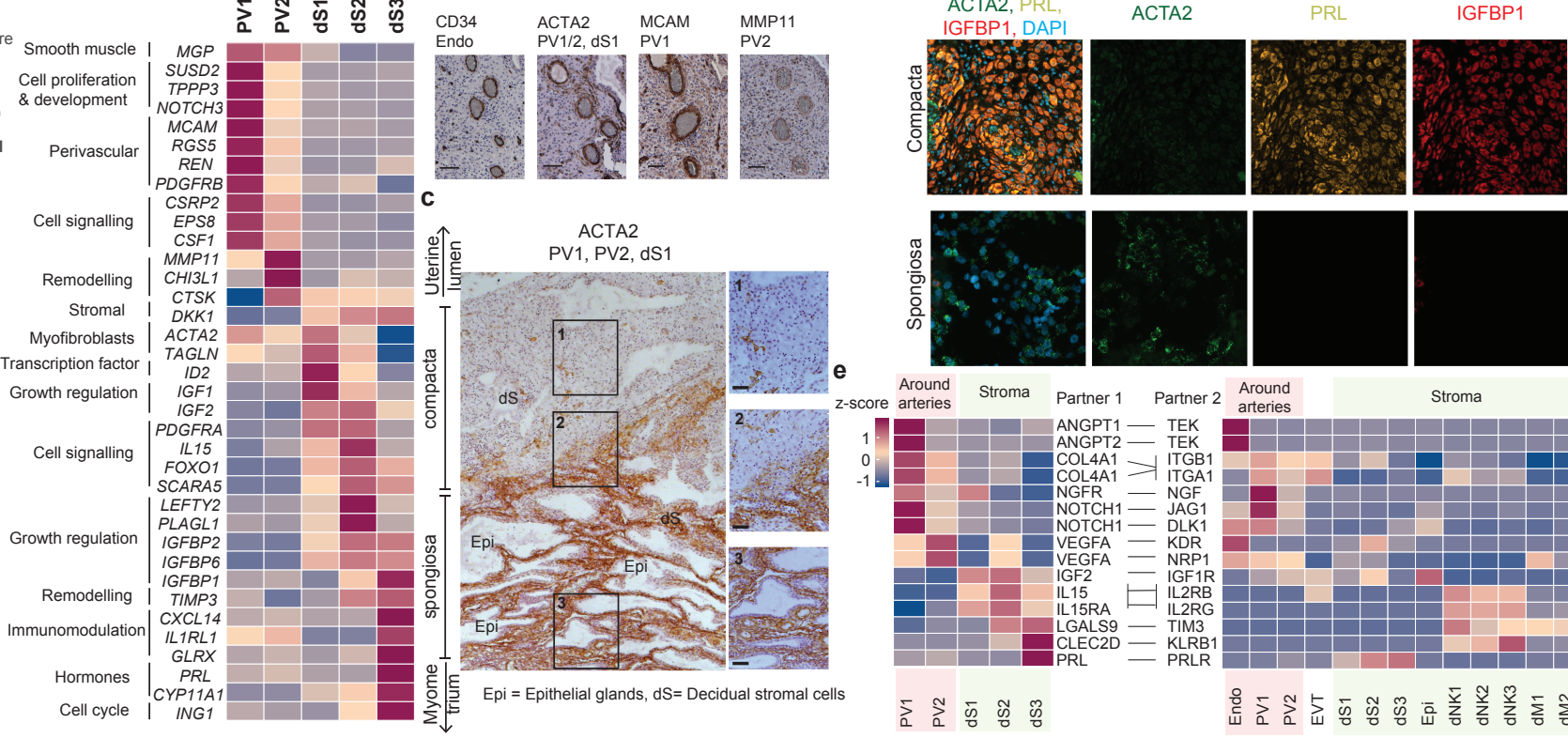

Transcription factor TAGLN

Growth regulation IGF1

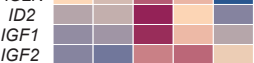

Cell signalling 1
IL15
FOXO1 FOXO1

LEFTY2

LEFTY2
PLAGL1

IGFBP2

IGFBP1

Remodelling 1 TIMP3

Immunomodulation $\begin{gathered}C X C L 14 \\ \text { IL1RL1 }\end{gathered}$

Hormones $\begin{array}{r}G L R X \\ P R L\end{array}$

$G L R X$
PRL
YP11A1
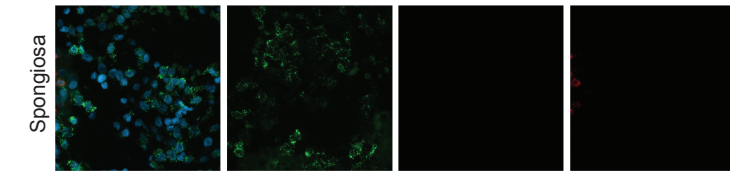

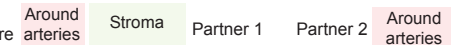

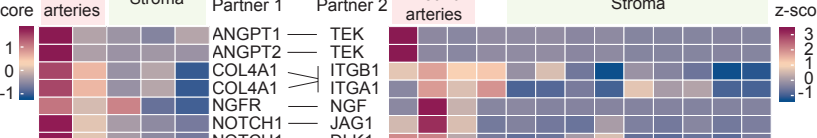

Cell cycle | ING

Epi $=$ Epithelial glands, $d S=$ Decidual stromal cells 


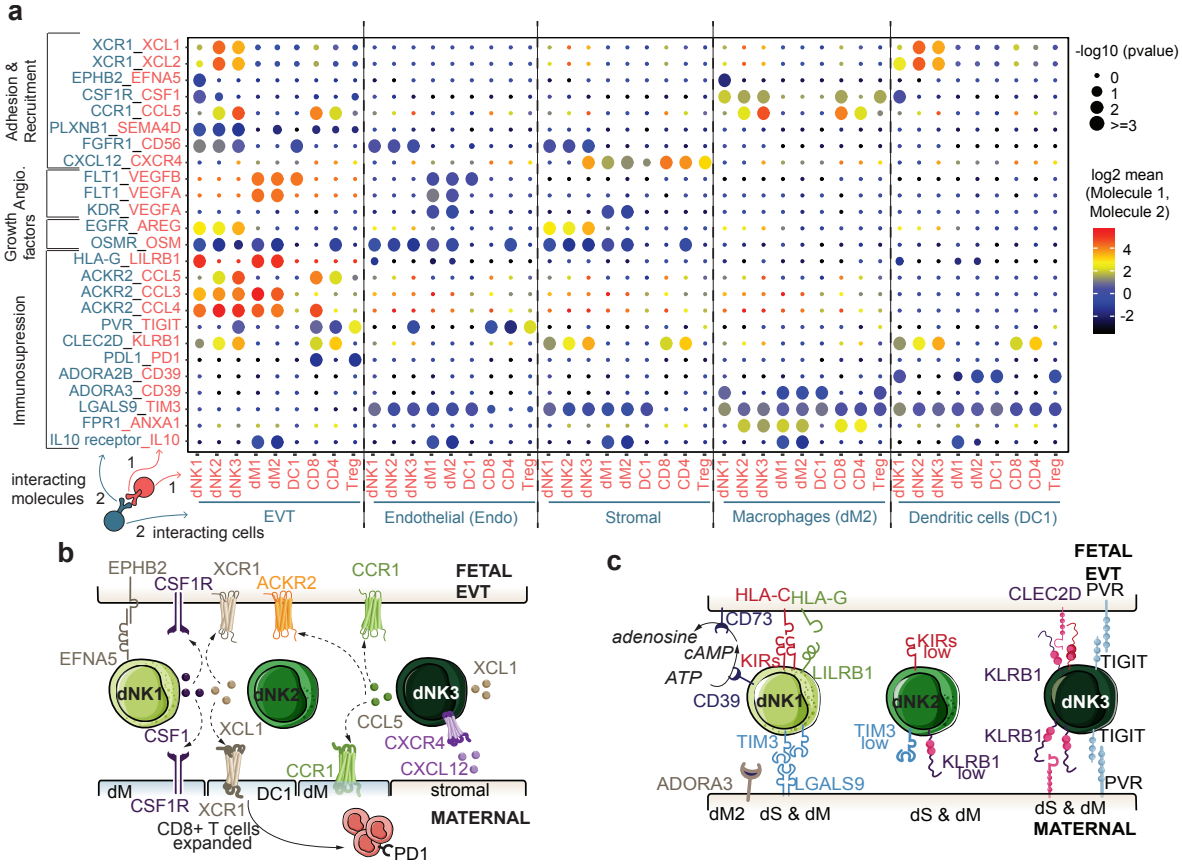

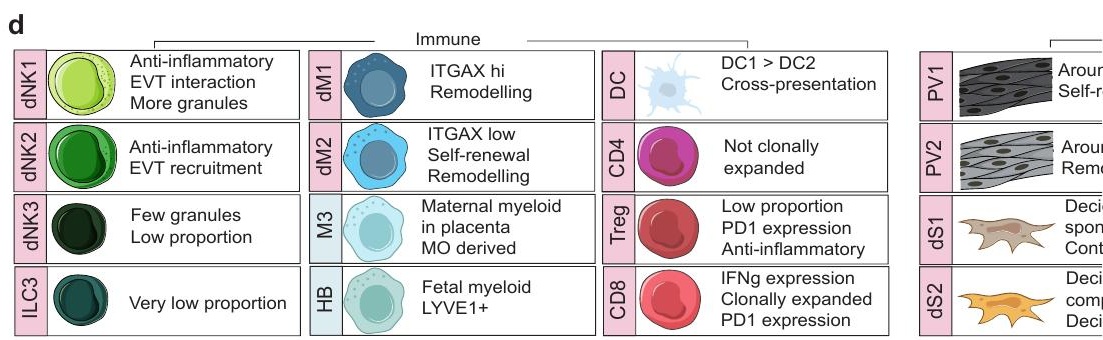


